\title{
A Novel APPROACH FOr SEgmentation OF SECTOR SCAN SONAR IMAGES USING ADAPTIVE THRESHOLDING
}

\author{
Nagamani Modalavalasa ${ }^{1}$, G SasiBhushana Rao ${ }^{2}$, K. Satya Prasad ${ }^{3}$ \\ ${ }^{1}$ Dept.of ECE, SBTET, Andhra Pradesh, INDIA \\ mani.modalavalasa@gmail.com \\ ${ }^{2}$ Dept. of ECE, Andhra University, Visakhapatnam, Andhra Pradesh, INDIA \\ ${ }^{3}$ Dept.of ECE, Jawaharlal Nehru Technological University Kakinada, Kakinada, INDIA
}

\begin{abstract}
The SAR and SAS images are perturbed by a multiplicative noise called speckle, due to the coherent nature of the scattering phenomenon. If the background of an image is uneven, the fixed thresholding technique is not suitable to segment an image using adaptive thresholding method. In this paper a new Adaptive thresholding method is proposed to reduce the speckle noise, preserving the structural features and textural information of Sector Scan SONAR (Sound Navigation and Ranging) images. Due to the massive proliferation of SONAR images, the proposed method is very appealing in under water environment applications. In fact it is a pre- treatment required in any SONAR images analysis system. The results obtained from the proposed method were compared quantitatively and qualitatively with the results obtained from the other speckle reduction techniques and demonstrate its higher performance for speckle reduction in the SONAR images.
\end{abstract}

\section{KEYWORDS}

Adaptive thresholding, SONAR, segmentation, morphological operations, edge detection.

\section{INTRODUCTION}

The SONAR systems exploits techniques developed in the radar field and the capabilities of the high resolution imagery in a great number of applications such as marine geology, Commercial fishing, offshore oil prospecting and drilling, cable and pipeline lying and maintenance and underwater warfare[1]. In particular SONAR images are potentially low quality images. Based on acquisition conditions, the Signal to Noise Ratio (SNR) can be very low. Another feature of the SONAR images is they contain almost homogeneous and textured regions. The presence of edges is relatively rare. Using an important number of transducers, operating at sound or ultrasound frequencies, the SONAR systems generate high resolution images. The user can observe, analyzing such an image, some regions. This observation process is perturbed by the speckle noise[2]. Speckle noise has a characteristic of a multiplicative noise[3]. Hence the original SONAR image may not be suitable for process and analysis using image processing techniques. The removal of noise in the SONAR image is still a challenging task for the researcher due to artifacts and causes blurring of the image. There is no unique technique for enhancement of images. Different algorithms are used depending on the model of the noise. Many methods have 
been proposed for image enhancement [4], [5], [6]. A survey of digital image enhancement techniques can be found in [7], [8], [9], [10]. Most of those methods are based on gray-level histogram modifications [9], [11], while other methods are based on local contrast transformation and edge analysis [12], [13], or the "global" entropy transformation [14]. In all of these methods, there are no general standards for image quality which could be used as a design criteria for image enhancement algorithms. Hence an efficient image enhancement technique is often necessary to take the first and foremost step for the removal of noise in the SONAR images before it can process and analyze. By keeping the time parameter as the constraint and also considering the noise characteristics of the SONAR images, we proposed an algorithm in which Adaptive thresholding with image histogram is made use of for segmentation of SONAR images.

To extract the object pixels from the background pixels in the images, image thresholding technique is one of the important tool in the image processing and computer vision. Most of the techniques uses statistical approach on the complete image that determines a single threshold called global threshold for a complete image or determining threshold for each window called local threshold on a small region window.

When the background is uneven as a result of poor or non-uniform conditions a fixed or global threshold will not segment the image correctly. Local threshold is the sophisticated method to create a threshold surface so that different thresholds could be used for each individual pixel in the image. In this method, first the image was divided into a regular grid of non-overlapping sub regions and a threshold was assigned to the center of each sub region which had bimodal histogram by a fixed thresholding method. Then the threshold surface was interpolated from these local threshold values. They assumed an individual object had a fixed threshold with respect to the background, but different objects may have different thresholds. Various gray-level thresholds were tried to segment the image and the segmented objects were validated by the gradients along their boundaries. In this paper new adaptive thresholding method is proposed in which the threshold is calculated by an iterative process. The initial threshold value for each individual region of image is selected by making use of the histogram of the image region.

\section{AlgorithM DESCRIPTION}

Adaptive thresholding changes the threshold dynamically over the image. Adaptive thresholding typically takes a gray scale or colour image as input and, in the simplest implementation, outputs a binary image representing the segmentation. For each region in the image, a threshold has to be calculated. If the pixel value in that region is below the threshold it is set to the background value, otherwise it assumes the foreground value. The more sophisticated version of thresholding can accommodate changing illuminating conditions in the image. This is also known as local or dynamic thresholding [15].

The histogram is a digital image with $\mathrm{L}$ total possible intensity levels in the range $[0, \mathrm{G}]$ is defined as the discrete function

$$
h\left(r_{k}\right)=n_{k}
$$

Where, $r_{k}$ is the $k$ th intensity level in the interval $[0, \mathrm{G}]$ and $n_{k}$ is the number of pixels in the image whose intensity level is $r_{k}[16]$.

The normalized histograms can be obtained simply by dividing all elements of $h\left(r_{k}\right)$ by the total number of pixels in the image, which is denoted by $n$ :

$$
p\left(r_{k}\right)=\frac{h\left(r_{k}\right)}{n_{k}}=\frac{n_{k}}{n} \text { for } k=1,2, \ldots . . \mathrm{L} .
$$


From basic probability, we recognize $p\left(r_{k}\right)$ as an estimate of the probability of occurrence of intensity level $r_{k}$.

Algorithm for Segmentation of the SONAR images is as follows:

1. Smooth the image by median filtering.

2. Partition the image into several regions.

3. For each individual region select the initial threshold value $T$ from the histogram of corresponding region [17].

4. Segment the region using $T$. This will produce two groups of pixels: the object pixels (G1) and background pixels (G2).

G1: Consists of all pixels with intensity values $\geq T$

G2: Consists of pixels with values $<T$.

5. Average intensity values of $\mathrm{G} 1$ and $\mathrm{G} 2$ are computed

$$
\begin{aligned}
& m_{1}=\text { mean }(\mathrm{G} 1) \\
& m_{2}=\text { mean }(\mathrm{G} 2)
\end{aligned}
$$

6. A new threshold is computed that is the average of $\mathrm{m} 1$ and $\mathrm{m} 2$.

$$
T=\frac{1}{2}\left(m_{1}+m_{2}\right)
$$

7. Go back to step 4, now using the new threshold computed in step 6, keep repeating the steps from 4 to 6 until convergence has been reached (i.e. difference in $T$ in successive iterations is smaller than a predefined parameter $T_{o}$ ).

8. Similar procedure is followed to segment each individual region and combined to get processed image to identify the possible objects present in the image.

9. The small background clusters still remained in the image are removed by the morphological operation "imopen" with suitable structuring element. In the present underwater image scenario, we have elected "line" as the structuring element [18, 19].

Fourth to sixth steps are the keys to this method. The choices of threshold is difficult to determine. These factors influence the positions of the boundary points and consequently affect the interpolation results.

\section{RESULTS AND DISCUSSIONS}

In this section, we will compare some experimental results of our proposed method with some other methods as shown in Table 1. All these experiments are implemented on Real SONAR images which have been acquired from Defence Research \& Development Organisation, Naval Science \& Technological Laboratory (NSTL), Visakhapatnam, by conducting experiments at the NSTL's Towing Tank. The sample real image on which analysis has been undertaken is shown in Figure 1 and the image after segmentation from the proposed method is shown in Figure 2. Images after segmentation by the remaining methods are also shown from Figure 3 to Figure 6 and the Figure caption itself indicates the method by which the result is obtained.

The results clearly demonstrate that the proposed method is far superior than existing methods such as canny edge detection, edge detection based on differencing technique, adaptive histogram equalization, FCM thresholding methods. All the methods except proposed method were detected false alarms along with the detected edges of the real object with discontinuities. The results show that our proposed method is better than traditional approaches for segmentation as 
the object detected found by the proposed method is very accurate and false targets are eliminated by the proposed technique.

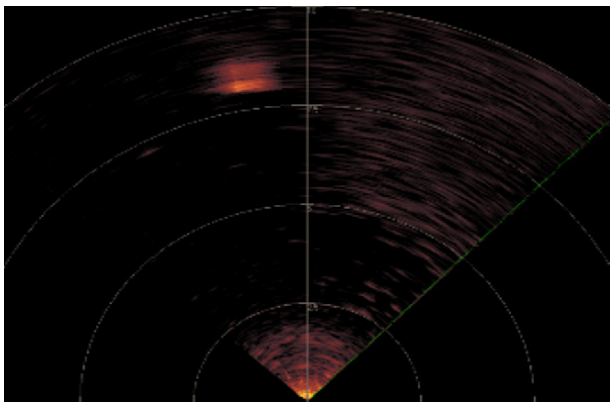

Figure 1. Real SONAR image

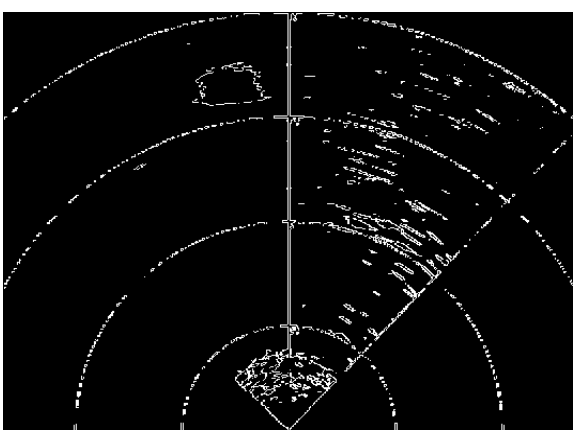

Figure 3. Edge detection based on differencing technique

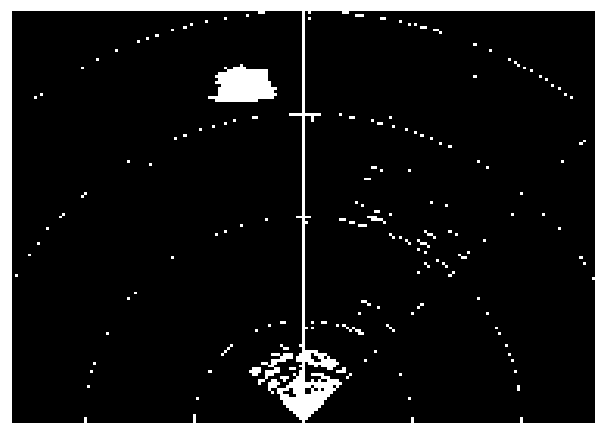

Figure 5. FCM Thresholding

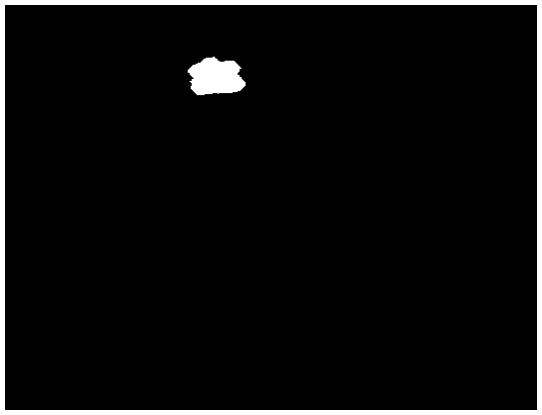

Figure 2. Object identification from the nronosed segmentation

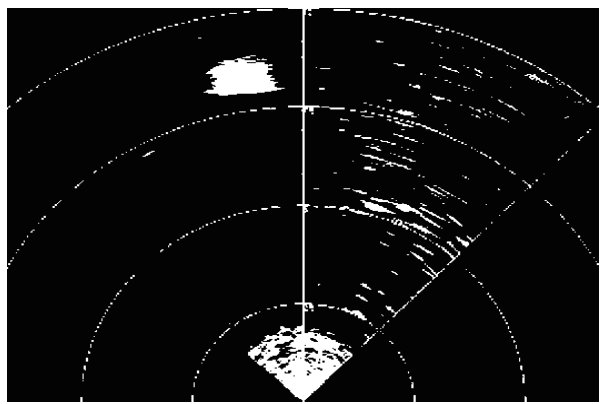

Figure 4. Adaptive Histogram Equalization

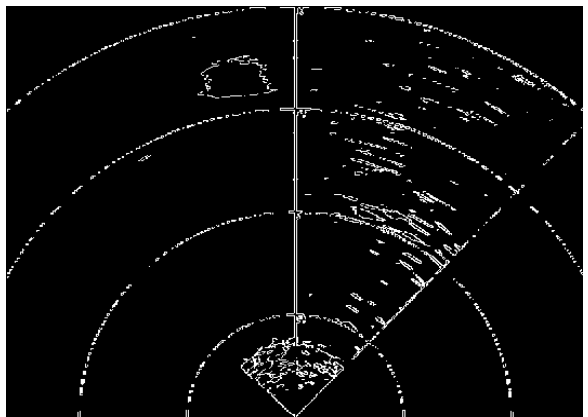

Figure 6.Canny Edge detection 
International Journal of Information Sciences and Techniques (IJIST) Vol.2, No.4, July 2012

Table 1. Performance comparison of proposed method with the other methods

\begin{tabular}{|l|l|l|l|}
\hline S.No. & Method & Execution Time (sec) & PSNR (dB) \\
\hline 1 & Canny Edge Detection & 1.109931 & 12.6053 \\
\hline 2 & $\begin{array}{l}\text { Edge detection based on } \\
\text { difference technique }\end{array}$ & 4.242526 & 13.4538 \\
\hline 3 & $\begin{array}{l}\text { Adaptive histogram } \\
\text { equalization }\end{array}$ & 2.1358 & 15.0972 \\
\hline 4 & FCM thresholding & 212.157879 & 9.6658 \\
\hline 5 & Proposed method & 0.171235 & 16.6109 \\
\hline
\end{tabular}

To identify better filtering algorithm for denoising of SONAR images a comparative study is done based on their error metrics. Two objective measures such as, MSE and PSNR are used for analyzing the performance of the filters.

a) Mean Square Error (MSE)

$$
M S E=\frac{1}{M N} \sum_{i=1}^{M} \sum_{j=1}^{N}\|I(i, j)-K(i, j)\|^{2}
$$

Where I $(i, j)$ denotes the samples of the original image and $K(i, j)$ denotes the samples of the filtered image. $M$ and $N$ are the number of pixels in row and column directions, respectively.

b) Peak Signal to Noise Ratio (PSNR)

$$
P S N R=10 \log 10\left(\frac{M A X_{I}^{2}}{M S E}\right)=20 \log 10\left(\frac{M A X}{\sqrt{M S E}}\right)
$$

Here, $M A X_{I}$ is the maximum pixel value of the original image. When the pixels are represented using 8 bits per sample, this value is 255 .

\section{Conclusions}

Automatic interpretation of SONAR images is extremely difficult because of the speckle noise present in them. This is the reason why the SONAR images denoising techniques were more and more interesting for the signal and image processing community till this day. This paper is concerned with the problem of speckle noise filtering in SONAR images using Adaptive thresholding method by finding the threshold adaptively for each sub region of the image followed by some morphological operations to identify the objects. The results of the proposed method have been compared with the existing methods. They are applied directly on real Sector Scan SONAR images. Both visual and numerical evaluations are given. It can be concluded that the proposed method gives better results by achieving high PSNR and low computing load i.e. less execution time. Hence, the proposed algorithm is best suited for segmenting the underwater SONAR images specifically for the application of obstacle avoidance by the AUV. 
International Journal of Information Sciences and Techniques (IJIST) Vol.2, No.4, July 2012

\section{ACKNOWLEDGEMENTS}

The above work has been undertaken towards the research project of DRDO (NSTL), Visakhapatnam, Andhra Pradesh, INDIA, No: NSTL/NA/9162/AUV/Consultancy/4 Dated $21 / 11 / 2007$. Authors are thankful to the management of their respective organizations. As well as grateful to my research guides, Dr. G. Sasi Bhushana Rao, Head of the Department, Electronics and Communication Engineering, Andhra University, Visakhapatnam, India and Dr. K. Satya Prasad, Rector, Jawharlal Nehru Technological University, Kakinada, India towards the Research Degree.

\section{REFERENCES}

[1] Laureant Hellequin, Jean-Marc Boucher, Xavier Lurton, "Processing of High-Frequency Muitibeam Echo Sounder for Seafloor Characterization", IEEE Joumal of Oceanic Engineering, vol. 28, no.1, pp. 78-89, January 2000.

[2] H. GUO, J E Odegard, M.Lang, R.A.Gopinath, I.W.Selesnick, and C.S. Burrus, "Wavelet based Speckle reduction with application to SAR based ATD/R", First Int'I Conf. on image processing, vol. 1, pp. 75-79,Nov 1994.

[3] Langis Ganon “Wavelet Filtering of Speckle Noise-Some Numerical Result," Proceeding of theConference Vision Interface 1999, Trois-Riveres.

[4] A. Rosenfeld and A. C. Kak, "Digital Picture Processing", New York: Academic, 1982, vol. 1.

[5] A. Jain, Fundamentals of Digital Image Processing. Englewood Cliffs, NJ: Prentice-Hall, 1989.

[6] T. L. Ji, M. K. Sundareshan, and H. Roehrig, "Adaptive image contrast enhancement based on human visual properties,” IEEE Trans. Med. Imag., vol. 13, pp. 573-586, Dec. 1994.

[7] S. S. Agaian, Advances and Problems of Fast Orthogonal Transform for Signal/Image Processing Applications, pp. 146-215, 1990.

[8] S. S. Agaian, Advances and Problems of Fast Orthogonal Transform for Signal/Image Processing Applications. Moscow, Russia: Nauka, 1991, pp. 99-145.

[9] S. Aghagolzadeh and O. K. Ersoy, "Transform image enhancement," Opt. Eng., vol. 31, pp. 614-626, Mar. 1992.

[10] D. Wang and A. H. Vagnucci, "Digital image enhancement," Comput. Vis., Graph., Image Process., vol. 24, pp. 363-381, 1981.

[11] W. M. Morrow, R. B. Paranjape, R. M. Rangayyan, and J. E. L. Desautels, "Region-based contrast enhancement of mammograms," IEEE Trans. Med.

[12] T. L. Ji, M. K. Sundareshan, and H. Roehrig, "Adaptive image contrast enhancement based on human visual properties,” IEEE Trans. Med. Imag., vol. 13, pp. 573-586, Dec. 1994.

[13] A. Beghcladi and A. L. Negrate, "Contrast enhancement technique based on local detection of edges," Comput. Vis., Graph., Image Process., vol. 46, pp. 162-274, 1989.

[14] A. M. Grigoryan and S. S. Agaian, "Split manageable efficient algorithm for Fourier and Hadamard transforms," IEEE Trans. Signal Processing, vol. 48, pp. 172-183, Jan. 2000.

[15] G. Martinez, "Criterion for automatic selection of the most suitable maximum-likelihood thresholding algorithm for extracting object from their background in a still image," in Proceedings of IAPR Conference on Machine Vision Applications, Tsukuba, Japan, May 16-18 2005.

[16] M. J. Carlotto, “Histogram analysis using a scale-space approach,' IEEE Trans. Pattern Anal. Mach. Intell. PAMI-9, 121-129 (1997).

[17] N. Ramesh, J. H. Yoo, and I. K. Sethi, “Thresholding based on histogram approximation," IEE Proc. Vision Image Signal Process. 142(5), 271-279 (1995).

[18] D. Anoraganingrum, "Cell segmentation with median filter and mathematical morphology operation," in Proceedings of IEEE International Conference on Image Analysis and Processing, Venice, Italy, September 27- 291999.

[19] I. Cseke, "A fast segementation scheme for white blood cells images," in Proceedings of IAPR International Conference on Pattern Recognition, Speech and Signal Analysis, Quebec, Canada, August 11-15 1992.

[20] Zhang, Y.J. and Luo, H.T. (2000): Optimal selection of segmentation algorithms based on performance evaluation. Optimal Engineering 3(96): 1450-1456. 


\section{Authors}

Smt Nagamani Modalavalasa received B.E. degree in Electronics and Communication Engineering from Andhra University, Visakhapatnam, Andhra Pradesh, India. She has completed her Master degree from Andhra University, Visakhapatnam, India. At present she is a research scholar in Electronics \& Communication Engg. Department, JNTU Engg. College, Kakinada, Andhra Pradesh, India. She has 15 years of teaching experience as Lecturer in the Department of Electronics \& Communication Engg, State Board of Technical Education \& Training, Andhra Pradesh, India. She has published around 18 research papers in various International, National conferences and journals.

Dr. G. Sasi Bhushana Rao received B E. degree in Electronics and Communication Engineering from Andhra University College of Engineering, Visakhapatnam, Andhra Pradesh, India, M.Tech. degree from JNTU, Hyderabad, India and Ph.D. from Osmania University, Hyderabad, India. He possesses vast administration, teaching and R\&D experience at Airports Authority, Andhra University, India for about 25 years. Currently he is working as Head Of Department in the Department of Electronics \& Communication Engg, Andhra University Engineering College, Visakhapatnam, India.

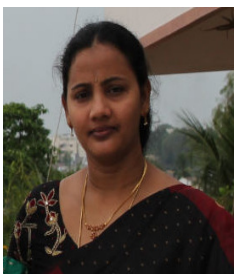
He has published more than 230 Technical and research papers in different National / International conferences and Journals and authored two Text books. He has guided 4 Ph.D. scholars and at present 14 scholars are working with him. His areas of Research include Inertial Navigation System (INS), GPS/GNSS Signal processing, Ionosphere/Troposphere and Multipath error modeling, and RADAR and SONAR navigation. Dr. Rao is a Fellow member of various professional bodies like IEEE, IETE, IGU, and International GNSS society.

Dr. K. Satya Prasad received B Tech. degree in Electronics and Communication Engineering from JNTU college of Engineering, Anantapur, Andhra Pradesh, India, M.E. degree in Communication Systems from Guindy college of Engg. , Madras University, Chennai, India and Ph. D from Indian Institute of Technology, Madras. He has more than 31 years of experience in teaching and 23 years of $\mathrm{R} \& \mathrm{D}$. He started his teaching carrier as Teaching Assistant at Regional Engineering College, Warangal in 1979. He joined JNT University, Hyderabad as Lecturer in 1980 and

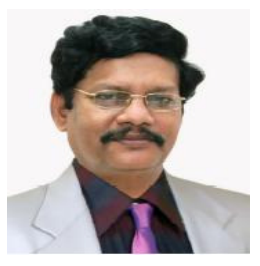
served in different constituent college's viz., Kakinada, Hyderabad and Anantapur and at different capacities viz., Associate Professor, Professor, and Head of the Department, Vice Principal and Principal. He has published more than 60 technical papers in different National / International conferences and Journals and authored one Text book. He has guided 4 Ph.D. scholars and at present 12 scholars are working with him. His areas of Research include Communications Signal Processing, Image Processing, Speech Processing, Neural Networks \& Ad-hoc wireless networks etc. Dr. Prasad is a Fellow member of various professional bodies like IETE, IE, and ISTE. 